\title{
Allylic Tantalums as Highly Imine-selective Reagents
}

\author{
Ikuya Shibata, ${ }^{*}$ Kyo Nose, Kazuya Sakamoto, Makoto Yasuda and Akio Baba* \\ Department of Molecular Chemistry, Science and Technology Center for Atoms, Molecules and Ions \\ Control, Graduate School of Engineering, Osaka University \\ 2-1 Yamadaoka, Suita, Osaka 5650871, Japan \\ shibata@chem.eng.osaka-u.ac.jp
}

\section{Supporting Information}

1. Experimental

1-1 General

S2

1-2 Sn-Ta exchange

1-3 Allylation of Imines

Representative Procedure of Allylation to $N$-Aromatic Imines.

Representative Procedure of One-pot Allylation using N-Aromatic Amines. $\quad$ S2

Representative Procedure of Allylation to $N$-Aliphatic Imines. $\quad$ S3

Representative Procedure of One-pot Allylation using $N$-Aliphatic Amines. $\quad \mathrm{S} 3$

2. Spectral data of products $\quad$ S4

Product 2a, 2b, 2c $\quad$ S4

Product 2d, 2e, $2 \mathrm{~g} \quad$ S5

Product 2i, 2k, 2l 2 S6

$\begin{array}{ll}\text { Product 2m, 2n, } 20 & \text { S7 }\end{array}$

$\begin{array}{ll}\text { Product } 2 \mathbf{p}, \mathbf{2 q}, \mathbf{2 r} & \text { S8 }\end{array}$

Product, $2 \mathbf{s}, \mathbf{2 t}, \mathbf{2 u} \quad$ S9

3. ${ }^{13} \mathrm{C}$ NMR Chart $\quad$ S10

$\begin{array}{ll}{ }^{13} \text { C NMR Chart 2b } & \text { S10 }\end{array}$

${ }^{13} \mathrm{C}$ NMR Chart 2c $\quad$ S11

$\begin{array}{ll}{ }^{13} \mathrm{C} \text { NMR Chart } 2 \mathbf{e} & \mathrm{S} 12\end{array}$

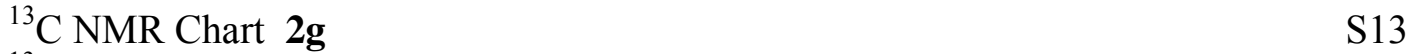

$\begin{array}{ll}{ }^{13} \text { C NMR Chart } \mathbf{2 k} & \text { S14 }\end{array}$

$\begin{array}{ll}{ }^{13} \mathrm{C} \text { NMR Chart 2l } & \mathrm{S} 15\end{array}$

$\begin{array}{ll}{ }^{13} \mathrm{C} \text { NMR Chart } 2 \mathrm{~m} & \text { S16 }\end{array}$

$\begin{array}{ll}{ }^{13} \mathrm{C} \text { NMR Chart 2n } & \text { S17 }\end{array}$

$\begin{array}{ll}{ }^{13} \mathrm{C} \text { NMR Chart } 2 \mathrm{~s} & \mathrm{~S} 18\end{array}$

$\begin{array}{ll}{ }^{13} \mathrm{C} \text { NMR Chart } 2 \mathrm{t} & \mathrm{S} 19\end{array}$

$\begin{array}{ll}{ }^{13} \mathrm{C} \text { NMR Chart } 2 \mathbf{u} & \text { S20 }\end{array}$ 


\section{Experimental}

\section{1-1 General}

Materials. Allyl-, crotyl-tri-n-butyltin were prepared by the reaction of tri- $n$-butyltin chloride with the

corresponding Grignard reagents. ${ }^{1}$ Commercially available $\mathrm{TaCl}_{5}$ was used. THF was freshly distilled from sodium benzophenone ketyl. All reactions were carried out under dry nitrogen.

1) Y. Naruta, Y. Nishigaichi, K. Maruyama, Chem. Lett. 1986, 1857.

\section{1-2 Sn-Ta exchange}

GLC analysis of Generated $n \mathrm{Bu}_{3} \mathrm{SnCl}$ : To a dry nitrogen-filled $10 \mathrm{~mL}$ round-bottomed flask containing $\mathrm{TaCl}_{5}(0.358 \mathrm{~g}, 1 \mathrm{mmol})$ in $\mathrm{CH}_{2} \mathrm{Cl}_{2}(1 \mathrm{~mL})$ was added allyltri-n-butyltin $(0.662 \mathrm{~g}, 2 \mathrm{mmol})$ at $-78{ }^{\circ} \mathrm{C}$. The resulting solution was measured by GLC analyses with SE-52. The peak of $n \mathrm{Bu}_{3} \mathrm{SnCl}$ was detected at $2.5 \mathrm{~min}$. Column temperature $180{ }^{\circ} \mathrm{C}$, flow pressure: $1.5 \mathrm{~kg} / \mathrm{cm}^{3}$, injector temp: $250{ }^{\circ} \mathrm{C}$, detector temp: $250{ }^{\circ} \mathrm{C}$.

NMR Measurement of Generated $n \mathrm{Bu}_{3} \mathrm{SnCl}$; Allyltri- $n$-butyltin $(0.331 \mathrm{~g}, 1 \mathrm{mmol})$ and $\mathrm{TaCl}_{5}(0.358 \mathrm{~g}$,

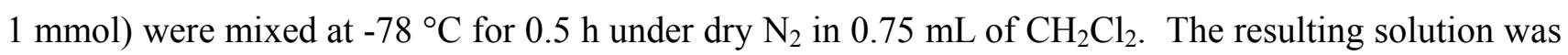
transferred to a nitrogen-filled $5 \mathrm{~mm} \phi \mathrm{NMR}$ tube, and the tube was fixed into a $10 \mathrm{~mm} \phi \mathrm{NMR}$ tube containing $\mathrm{CDCl}_{3}$ solution of $\mathrm{Me}_{4} \mathrm{Sn}$ as external standard, and the $10 \mathrm{~mm} \phi$ tube was set in the NMR instrument. Measurement was performed at $-78{ }^{\circ} \mathrm{C} . n \mathrm{Bu}_{3} \mathrm{SnCl}$ was detected at $123 \mathrm{ppm}$, and starting allyltri- $n$-butyltin was not detected at all.

\section{1-3 Allylation of Imines}

\section{Representative Procedure of Allylation to $\mathrm{N}$-Aromatic Imines.}

To a dry nitrogen-filled $10 \mathrm{~mL}$ round-bottomed flask containing $\mathrm{TaCl}_{5}(0.358 \mathrm{~g}, 1 \mathrm{mmol})$ in $\mathrm{CH}_{2} \mathrm{Cl}_{2}$ $(1 \mathrm{~mL})$ was added allyltri-n-butyltin $(0.662 \mathrm{~g}, 2 \mathrm{mmol})$ at $-78{ }^{\circ} \mathrm{C} . \mathrm{TaCl}_{5}$ was partly insoluble. After stirring at $-78{ }^{\circ} \mathrm{C}$ for $30 \mathrm{~min}$, to the resulting solution was added benzylideneaniline (1a) $(0.181 \mathrm{~g}, 1$ mmol). As the reaction proceeded, the mixture gradually turned homogeneous. During the reaction, the solution indicated a slight pale yellow color. After stirring the mixture at $-78{ }^{\circ} \mathrm{C}$ for $2 \mathrm{~h}$, the reaction mixture was quenched by saturated $\mathrm{NaHCO}_{3}(2 \mathrm{~mL})$. To the mixture were added saturated $\mathrm{NH}_{4} \mathrm{~F}(2 \mathrm{~mL})$ and ether $(10 \mathrm{~mL})$. After stirring at $\mathrm{rt}$ for $30 \mathrm{~min}$, the precipitated $\mathrm{Bu}_{3} \mathrm{SnF}$ was filtered off and the filtrate was extracted with ether $\left(15 \mathrm{~mL}\right.$ x 3), dried over $\mathrm{MgSO}_{4}$ and filtered. Volatiles were removed under reduced pressure. The residue was chromatographed on silica-gel column (FL100-DX (Fuji silysia)), eluting with hexane/EtOAc (99/1) to give homoallylamine 2a (0.205 g, 92\%).

\section{Representative Procedure of One-pot Allylation using $N$-Aromatic Amines.}

To a dry nitrogen-filled $10 \mathrm{~mL}$ round-bottomed flask containing $\mathrm{TaCl}_{5}(0.358 \mathrm{~g}, 1 \mathrm{mmol})$ in THF (1 $\mathrm{mL})$ was added allyltri-n-butyltin $(0.331 \mathrm{~g}, 1 \mathrm{mmol})$ at $-78{ }^{\circ} \mathrm{C}$. After $30 \mathrm{~min}$, aniline $(0.093 \mathrm{~g})$ and 3 Phenylpropionaldehyde $(0.134 \mathrm{~g}, 1 \mathrm{mmol})$ were added and stirred the mixture at $-78{ }^{\circ} \mathrm{C}$ for $3 \mathrm{~h}$. The 
reaction mixture was quenched by saturated $\mathrm{NaHCO}_{3}(2 \mathrm{~mL})$. To the mixture were added saturated $\mathrm{NH}_{4} \mathrm{~F}(2 \mathrm{~mL})$ and ether $(10 \mathrm{~mL})$. After stirring at $\mathrm{rt}$ for $30 \mathrm{~min}$, the precipitated $\mathrm{Bu}_{3} \mathrm{SnF}$ was filtered off and the filtrate was extracted with ether $\left(15 \mathrm{~mL}\right.$ x 3), dried over $\mathrm{MgSO}_{4}$ and filtered. Volatiles were removed under reduced pressure. The residue was chromatographed on silica-gel column (FL100-DX (Fuji silysia)) eluting with hexane/EtOAc (99/1) to give homoallylamine $2 \mathbf{e}(0.249 \mathrm{~g}, 99 \%)$.

\section{Representative Procedure of Allylation to $\mathrm{N}$-Aliphatic Imines.}

To a dry nitrogen-filled $10 \mathrm{~mL}$ round-bottomed flask containing $\mathrm{TaCl}_{5}(0.358 \mathrm{~g}, 1 \mathrm{mmol})$ in $\mathrm{CH}_{2} \mathrm{Cl}_{2}$ $(1 \mathrm{~mL})$ were added $\mathrm{MgO}(0.201 \mathrm{~g}, 5 \mathrm{mmol})$ at $\mathrm{rt}$ and stirred for $1 \mathrm{~h}$. The solution was cooled to $-40{ }^{\circ} \mathrm{C}$, and allyltri-n-butyltin $(0.662 \mathrm{~g}, 2 \mathrm{mmol})$ was added. After stirring for $30 \mathrm{~min}$, to the resulting solution was added benzylide $N$-methylamine $(0.119 \mathrm{~g}, 1 \mathrm{mmol})$. After stirring the mixture at $-40{ }^{\circ} \mathrm{C}$ for $3 \mathrm{~h}$, the reaction mixture was quenched by saturated $\mathrm{NaHCO}_{3}(2 \mathrm{~mL})$. To the mixture were added saturated $\mathrm{NH}_{4} \mathrm{~F}(2 \mathrm{~mL})$ and ether $(10 \mathrm{~mL})$. After stirring at $\mathrm{rt}$ for $30 \mathrm{~min}$, the precipitated $\mathrm{Bu}_{3} \mathrm{SnF}$ was filtered off and the filtrate was extracted with ether $(15 \mathrm{~mL} \times 3)$. The organic layer was extracted with aqueous 0.5 $\mathrm{M} \mathrm{HCl}(15 \mathrm{~mL} \times 3)$, and products were transfered to a water layer as quaternary ammonium salts. To the water layer was added saturated $\mathrm{NaHCO}_{3}(10 \mathrm{~mL})$ slowly. The product was extracted with ether $(20$ mLx3), dried over $\mathrm{MgSO}_{4}$ and filtrated. Volatiles were removed under reduced pressure. The residue was purified by bulb-to-bulb distillation to give 2 n $(0.127 \mathrm{~g}, 79 \%)$.

\section{Representative Procedure of One-pot Allylation using N-Aliphatic Amines.}

To a dry nitrogen-filled $10 \mathrm{~mL}$ round-bottomed flask containing $n$-propylamine $(0.059 \mathrm{~g}, 1 \mathrm{mmol})$ and benzaldehyde $(0.106 \mathrm{~g}, 1 \mathrm{mmol})$ was added MS4A $(0.3 \mathrm{~g})$. To prepare the corresponding imine in situ, the mixture was stirred at rt for $15 \mathrm{~h}$. Allyltri-n-butyltin $(0.662 \mathrm{~g}, 2 \mathrm{mmol})$ and $\mathrm{MgO}(0.201 \mathrm{~g}, 5$ mmol) were added, and the solution was cooled to $-40{ }^{\circ} \mathrm{C}$. To the resulting solution was added $\mathrm{TaCl}_{5}$ $(0.358 \mathrm{~g}, 1 \mathrm{mmol})$. After stirring for $3 \mathrm{~h}$, the reaction mixture was quenched by saturated $\mathrm{NaHCO}_{3}(2$ $\mathrm{mL})$. Saturated $\mathrm{NH}_{4} \mathrm{~F}(2 \mathrm{~mL})$ and ether $(10 \mathrm{~mL})$ were added. After stirring at $\mathrm{rt}$ for $30 \mathrm{~min}$, the precipitated $\mathrm{Bu}_{3} \mathrm{SnF}$ was filtered off, and the filtrate was extracted with ether $(15 \mathrm{~mL} \times 3)$. The organic layer was extracted with $0.5 \mathrm{M} \mathrm{HCl}(15 \mathrm{~mL}$ x 3) when products were transfered to water layer as quaternary ammonium salts. To the water layer was added saturated $\mathrm{NaHCO}_{3}(10 \mathrm{~mL})$ slowly. The product was extracted with ether $\left(20 \mathrm{~mL}\right.$ x 3), dried over $\mathrm{MgSO}_{4}$ and filtered. Volatiles were removed under reduced pressure. The residue was purified by bulb-to bulb distillation to give $2 \mathbf{p}(0.134 \mathrm{~g}, 71 \%)$. 


\section{Spectral data of products}

(2a)

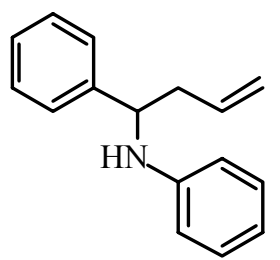

A. Studer, S. Amrein, Synthesis 2002, 928.

colorless wax; IR (neat) $3409 \mathrm{~cm}^{-1}$; ${ }^{1} \mathrm{H}-\mathrm{NMR}\left(\mathrm{CDCl}_{3}\right) \delta 2.41-2.64(\mathrm{~m}, 2 \mathrm{H}), 4.13(\mathrm{~s}, 1 \mathrm{H}), 4.37$ (dt, $J=$ $5.37,7.81 \mathrm{~Hz}, 1 \mathrm{H}), 5.13(\mathrm{dd}, J=9.77$ and $<1 \mathrm{~Hz}, 1 \mathrm{H}), 5.17(\mathrm{dd}, J=17.09$ and $<1 \mathrm{~Hz}, 1 \mathrm{H}$ ), 5.75 (ddt, $J=$ 17.09, 9.77, 7.32 Hz, 1H), 6.48 (d, J=7.33 Hz, 2H), 6.63 (t, J=7.33 Hz,1H), 7.01-7.38 (m, 7H). ${ }^{13} \mathrm{C}-$ NMR $\left(\mathrm{CDCl}_{3}\right) \delta 43.3,57.1,113.4,117.3,118.2,126.3,128.5,129.0,126.9-134.6$; HRMS calcd for $\mathrm{C}_{16} \mathrm{H}_{17} \mathrm{~N}$ 223.1361: Found 223.1364.

(2b)

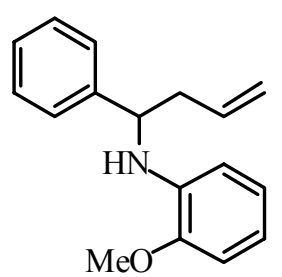

colorless wax; IR (neat) 3424, 1596, $1227 \mathrm{~cm}^{-1}$; ${ }^{1} \mathrm{H}-\mathrm{NMR}\left(\mathrm{CDCl}_{3}\right) \delta 2.49-2.63(\mathrm{~m}, 2 \mathrm{H}), 3.87(\mathrm{~s}, 3 \mathrm{H})$, $4.37(\mathrm{t}, J=7.33,1 \mathrm{H}), 4.79(\mathrm{~s}, 1 \mathrm{H}), 5.11-5.21(\mathrm{~m}, 2 \mathrm{H}), 5.70-5.86(\mathrm{~m}, 1 \mathrm{H}), 6.29$ (dd, J= 7.50, $1.46 \mathrm{~Hz}$, 2H), 6.56-6.77 (m, 3H), 7.19-7.37 (m, 5H). ${ }^{13} \mathrm{C}-\mathrm{NMR}\left(\mathrm{CDCl}_{3}\right) \delta 43.3$, 55.5, 57.2, 109.3, 111.1, 116.4, 118.0, 121.1, 126.3, 126.9, 128.5, 134.7, 137.2, 143.7, 146.8; HRMS calcd for $\mathrm{C}_{17} \mathrm{H}_{19} \mathrm{NO} 253.1467$ : Found 253.1458.

(2c)

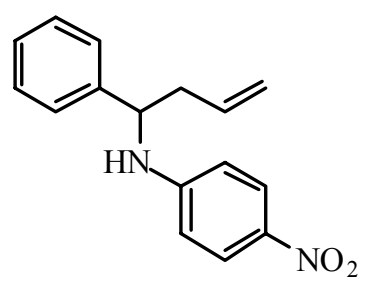

colorless wax; IR (neat) $3348 \mathrm{~cm}^{-1} ;{ }^{1} \mathrm{H}-\mathrm{NMR}\left(\mathrm{CDCl}_{3}\right) \delta 2.47-2.76(\mathrm{~m}, 2 \mathrm{H}), 4.52(\mathrm{dd}, J=5.37$ and 7.81 Hz, 1H) 4.87-4.97 (br, 1H), 5.16-5.29 (m, 2H), 5.64-5.83 (m, 1H), 6.44 (d, J=9.28 Hz, 2H), 7.20-7.41 $(\mathrm{m}, 5 \mathrm{H}), 7.99(\mathrm{~d}, J=9.18 \mathrm{~Hz}, 2 \mathrm{H}) .{ }^{13} \mathrm{C}-\mathrm{NMR}\left(\mathrm{CDCl}_{3}\right) \delta 42.8,56.6,112.1,119.2,126.0,126.1,127.6$, 128.90 133.6, 138.3, 141.4, 152.2; HRMS calcd for $\mathrm{C}_{16} \mathrm{H}_{16} \mathrm{~N}_{2} \mathrm{O}_{2}$ 268.1212: Found 268.1211; Anal calcd for $\mathrm{C}_{16} \mathrm{H}_{16} \mathrm{~N}_{2} \mathrm{O}_{2}$ : C, 71.62; H, 6.01, N,10.44: Found C, 71.80, H, 6.17, N, 10.33. 
(2d)

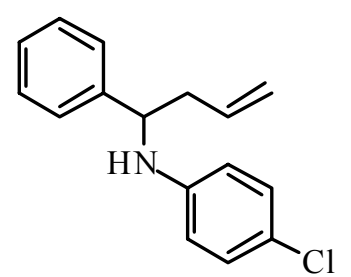

K. Manabe, Y. Mori, S. Kobayashi, Tetrahedron 2001, 57, 2537.

colorless wax; IR (neat) $3417 \mathrm{~cm}^{-1}$; ${ }^{1} \mathrm{H}-\mathrm{NMR}\left(\mathrm{CDCl}_{3}\right) \delta 2.40-2.68$ (m, $2 \mathrm{H}$ ), 4.10-4.22 (d, J=4.89 Hz, $1 \mathrm{H}), 4.33(\mathrm{dd}, J=4.89$ and $7.08 \mathrm{~Hz}, 1 \mathrm{H}), 5.08-5.25(\mathrm{~m}, 2 \mathrm{H}), 5.65-5.84(\mathrm{~m}, 1 \mathrm{H}), 6.36-6.46(\mathrm{~m}, 2 \mathrm{H}), 6.96-$ $7.04(\mathrm{~m}, 2 \mathrm{H}), 7.17-7.38(\mathrm{~m}, 5 \mathrm{H}) .{ }^{13} \mathrm{C}-\mathrm{NMR}\left(\mathrm{CDCl}_{3}\right)$ 843.2, 57.1, 114.5, 118.5, 121.9, 126.2, 127.1, 128.6, 128.9, 134.4, 143.0, 145.8; HRMS calcd for $\mathrm{C}_{16} \mathrm{H}_{16} \mathrm{ClN}$ 257.0971: Found 257.0962; Anal calcd for $\mathrm{C}_{16} \mathrm{H}_{16} \mathrm{ClN}: \mathrm{C}, 74.45 ; \mathrm{H}, 6.26, \mathrm{~N}, 5.43, \mathrm{Cl}, 13.75$ : Found C, 74.40, H, 6.33, N, 5.41, Cl, 13.53 .

(2e)

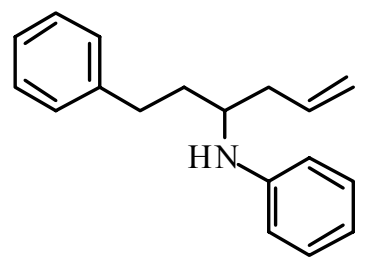

colorless wax; IR (neat) $3406 \mathrm{~cm}^{-1}$; ${ }^{1} \mathrm{H}-\mathrm{NMR}\left(\mathrm{CDCl}_{3}\right) \delta 1.64-1.93(\mathrm{~m}, 2 \mathrm{H}), 2.28(\mathrm{t}, J=6.35 \mathrm{~Hz}, 2 \mathrm{H})$, 2.59-2.79 (m, 2H), 3.35-3.60 (m, 2H), 4.99-5.11 (m, 2H), 5.68-5.86 (m, 1H), $6.51(\mathrm{~d}, J=7.81 \mathrm{~Hz}, 2 \mathrm{H})$, 6.65 (t, $J=7.32 \mathrm{~Hz}, 1 \mathrm{H}), 7.05-7.31(\mathrm{~m}, 7 \mathrm{H}) .{ }^{13} \mathrm{C}-\mathrm{NMR}\left(\mathrm{CDCl}_{3}\right) 832.2,35.9,38.4,51.6,113.3,117.1$, 117.7, 125.9, 128.4, 128.5, 129.3, 134.6, 1412.0, 147.6; HRMS calcd for $\mathrm{C}_{18} \mathrm{H}_{21} \mathrm{~N}$ 251.1674: Found 251.1683; Anal calcd for $\mathrm{C}_{18} \mathrm{H}_{21} \mathrm{~N}$ : C, 86.01; H, 8.42, N,5.57: Found C, 85.72, H, 8.53, N, 5.56.

(2g)

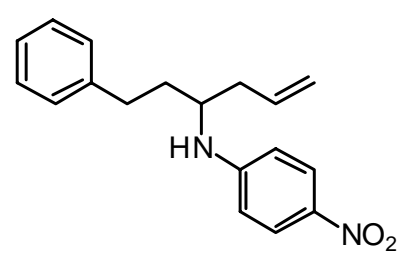

IR (neat) $3363 \mathrm{~cm}^{-1}$; ${ }^{1} \mathrm{H}-\mathrm{NMR}\left(\mathrm{CDCl}_{3}\right) \delta 1.78-1.99(\mathrm{~m}, 2 \mathrm{H}), 2.28-2.45(\mathrm{~m}, 2 \mathrm{H}), 2.69-2.76(\mathrm{~m}, 2 \mathrm{H})$, 3.51-3.61 (m, 1H), 4.39-4.44 (m, 1H), 5.08-5.16 (m, 2H), 5.70-5.85 (m,1H), 6.40-6.46 (m, 2H), 7.13$7.31(\mathrm{~m}, 5 \mathrm{H}), 8.03-8.08(\mathrm{~m}, 2 \mathrm{H}) .{ }^{13} \mathrm{C}-\mathrm{NMR}\left(\mathrm{CDCl}_{3}\right) 32.2,35.8,38.6,51.5,111.3,118.6,126.1,126.4$, 128.3, 128.5, 133.5, 137.6, 141.0, 152.7; HRMS calcd for $\mathrm{C}_{18} \mathrm{H}_{20} \mathrm{~N}_{2} \mathrm{O}_{2}$ 296.1525: Found. 296.1530. 
(2i)<smiles>C=CCC(Nc1ccccc1)C1CCCCC1</smiles>

S. Kobayashi; S. Nagayama, J. Am. Chem. Soc. 1997, 119, 10049.

${ }^{1} \mathrm{H}-\mathrm{NMR}\left(\mathrm{CDCl}_{3}\right)$ 80.84-1.85 (m, 11H), 2.14-2.39 (m, 2H), $3.24(\mathrm{dd}, J=5.1,6.9 \mathrm{~Hz}, 1 \mathrm{H}), 3.50$ (brs, 1H), 5.00-5.08 (m, 2H), 5.72-5.87 (m, 1H), 6.53-6.65 (m, 3H), 7.10-7.19 (m, 2H). ${ }^{13} \mathrm{C}-\mathrm{NMR}\left(\mathrm{CDCl}_{3}\right)$ $\delta 26.3,26.4,26.5,29.4,35.8,41.2,57.2,112.9,116.5,117.0,129.2,135.6,148.3$; HRMS calcd for $\mathrm{C}_{18} \mathrm{H}_{23} \mathrm{~N} 229.1877$ : Found 229.1854.

(2k)

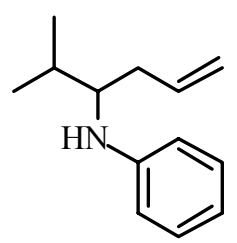

colorless wax; IR (neat) $3410 \mathrm{~cm}^{-1} ;{ }^{1} \mathrm{H}-\mathrm{NMR}\left(\mathrm{CDCl}_{3}\right) \delta 0.94$ (d, $\left.J=6.84 \mathrm{~Hz}, 3 \mathrm{H}\right), 0.97(\mathrm{~d}, J=6.84 \mathrm{~Hz}$, $3 \mathrm{H}), 1.90$ (dq, $J=13.64,6.84 \mathrm{~Hz}, 1 \mathrm{H}), 2.10-2.40$ (m, 2H), 3.19-3.32 (m, 1H), 3.52 (brs, $1 \mathrm{H}), 4.99-5.13$ $(\mathrm{m}, 2 \mathrm{H}), 5.71-5.90(\mathrm{~m}, 1 \mathrm{H}), 6.49-6.68(\mathrm{~m}, 3 \mathrm{H}), 7.08-7.19(\mathrm{~m}, 2 \mathrm{H}) .{ }^{13} \mathrm{C}-\mathrm{NMR}\left(\mathrm{CDCl}_{3}\right) \delta 18.3,18.5,30.7$, 35.52, 57.8, 113.1, 116.7, 117.1, 129.4, 135.8, 148.4; HRMS calcd for $\mathrm{C}_{18} \mathrm{H}_{23} \mathrm{~N}$ 189.1517: Found 189.1512 .

(2l)<smiles>C=CC(C)C(CCc1ccccc1)Nc1ccccc1</smiles>

colorless wax; IR (neat) $3402 \mathrm{~cm}^{-1}$; ${ }^{1} \mathrm{H}-\mathrm{NMR}\left(\mathrm{CDCl}_{3}\right)$ (major isomer) $\delta 1.00(\mathrm{~d}, 3 \mathrm{H}, J=7.01 \mathrm{~Hz}), 1.42-$ $1.71(\mathrm{~m}, 1 \mathrm{H}), 1.77-2.00(\mathrm{~m}, 1 \mathrm{H}), 2.49$ (qdd, $J=7.01,8.06$ and $4.64 \mathrm{~Hz}, 1 \mathrm{H}), 2.56-2.87(\mathrm{~m}, 2 \mathrm{H}), 3.22-$ $3.42(\mathrm{~m}, 1 \mathrm{H}), 3.51$ (brs, $1 \mathrm{H}), 4.91-5.12(\mathrm{~m}, 2 \mathrm{H}), 5.71$ (ddd, $J=17.55,10.37$ and $8.06 \mathrm{~Hz}, 1 \mathrm{H}), 6.48-6.70$ $(\mathrm{m}, 3 \mathrm{H}), 7.07-7.32(\mathrm{~m}, 7 \mathrm{H}) .{ }^{13} \mathrm{C}-\mathrm{NMR}\left(\mathrm{CDCl}_{3}\right)$ (minor) $815.09,32.75,34.00,40.27,56.45,112.97$, $115.28,116.71,125.70,128.24,128.37,129.20,140.24,141.99$, 148.07 (major) $\delta 16.7,32.8,33.2$, 41.5, $56.8,112.4,115.4,116.9,125.7,128.2,128.4,129.2$, 140.4, 142.1, 148.1; HRMS calcd for $\mathrm{C}_{19} \mathrm{H}_{23} \mathrm{~N}$ 265.1830: Found 265.1827; Anal calcd for $\mathrm{C}_{19} \mathrm{H}_{23} \mathrm{~N}$ : C, 85.99, H, 8.74, N, 5.28. Found C, 85.72, H, 8.75, N, 5.01. 
(2m)

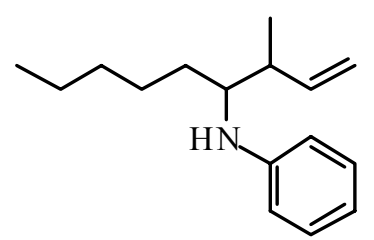

colorless wax; IR (neat) $3410 \mathrm{~cm}^{-1} ;{ }^{1} \mathrm{H}-\mathrm{NMR}\left(\mathrm{CDCl}_{3}\right)$ (major isomer) $\delta 0.78(\mathrm{t}, J=7.57 \mathrm{~Hz}, 3 \mathrm{H}), 1.05$ (d, $J=6.84 \mathrm{~Hz}, 3 \mathrm{H}), 1.16-1.68$ (m, 9H), 3.21-3.32 (m, 1H), 3.50 (brs, $1 \mathrm{H}), 4.92-5.13(\mathrm{~m}, 2 \mathrm{H}), 5.65-5.93$ $(\mathrm{m}, 1 \mathrm{H}), 6.51-6.67(\mathrm{~m}, 3 \mathrm{H}), 7.08-7.19(\mathrm{~m}, 2 \mathrm{H}) .{ }^{13} \mathrm{C}-\mathrm{NMR}\left(\mathrm{CDCl}_{3}\right)$ (major) $\delta 14.1,16.5,22.6,26.3,31.5$, 32.0, 41.4, 57.5, 113.12, 115.0, 116.6, 129.2, 140.8, 148.3; HRMS calcd for $\mathrm{C}_{16} \mathrm{H}_{25} \mathrm{~N}$ 231.1987: Found 231.1989 .

(2n)<smiles>C=CC(C)C(Nc1ccccc1)C1CCCCC1</smiles>

colorless wax; IR (neat) $3410 \mathrm{~cm}^{-1}$; ${ }^{1} \mathrm{H}-\mathrm{NMR}\left(\mathrm{CDCl}_{3}\right)$ (major isomer) $\delta 1.00(\mathrm{~d}, J=6.83 \mathrm{~Hz}, 3 \mathrm{H}), 0.88$ $1.34(\mathrm{~m}, 5 \mathrm{H}), 1.45-1.92(\mathrm{~m}, 6 \mathrm{H}), 2.34-2.53(\mathrm{~m}, 1 \mathrm{H}), 3.11$ (t, J=6.34 Hz, 1H), 3.40 (br, 1H), 4.90-5.13 $(\mathrm{m}, 2 \mathrm{H}), 5.68-5.90(\mathrm{~m}, 1 \mathrm{H}), 6.50-6.72(\mathrm{~m}, 3 \mathrm{H}), 7.05-7.23(\mathrm{~m}, 2 \mathrm{H}) .{ }^{13} \mathrm{C}-\mathrm{NMR}\left(\mathrm{CDCl}_{3}\right)$ (major) $\delta 16.4$, 26.3-26.6(3C), 27.8, 31.4, 41.3-41.4(2C), 62.1, 112.5, 113.8, 116.1, 129.1, 142.7, 149.7; HRMS calcd for $\mathrm{C}_{17} \mathrm{H}_{25} \mathrm{~N}$ 243.1987: Found 243.1982.

(20)<smiles>C=CCC(NC)c1ccccc1</smiles>

D-K. Wang, Y-G. Zhou, Y. Tang, X-L. Hou, L-X. Dai, J. Org. Chem. 1999, 64, 4233.

colorless wax; IR(neat) $3340 \mathrm{~cm}^{-1} ;{ }^{1} \mathrm{H}-\mathrm{NMR}\left(\mathrm{CDCl}_{3}\right) \delta 1.86(\mathrm{~s}, 1 \mathrm{H}), 2.26(\mathrm{~s}, 3 \mathrm{H}), 2.39-2.44(\mathrm{~m}, 2 \mathrm{H})$, $3.53(\mathrm{t}, J=6.35 \mathrm{~Hz}, 1 \mathrm{H}), 5.03-5.12(\mathrm{~m}, 2 \mathrm{H}), 5.62-5.80(\mathrm{~m}, 1 \mathrm{H}), 7.17-7.40(\mathrm{~m}, 5 \mathrm{H}) .{ }^{13} \mathrm{C}-\mathrm{NMR}\left(\mathrm{CDCl}_{3}\right)$ 834.4, 42.7, 64.5, 117.4, 126.9, 127.0, 128.2, 135.3, 143.3; HRMS calcd for $\mathrm{C}_{11} \mathrm{H}_{15} \mathrm{~N}$ 161.1204: Found 162.1277(CI, $\left.\left(\mathrm{M}^{+}\right)+1\right)$. 
(2p)

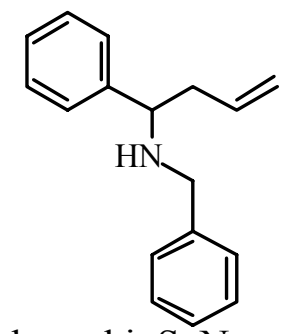

S. Kobayashi; S. Nagayama, J. Am. Chem. Soc. 1997, 119, 10049.

colorless wax; ${ }^{1} \mathrm{H}-\mathrm{NMR}\left(\mathrm{CDCl}_{3}\right) \delta 1.77$ (brs, $\left.1 \mathrm{H}\right), 2.41-2.46$ (m, 3H), 3.53 (d, J=13.4 Hz, 1H), 3.67 (d, $J=13.7 \mathrm{~Hz}, 1 \mathrm{H}), 5.02-5.11(\mathrm{~m}, 2 \mathrm{H}), 5.65-5.75(\mathrm{~m}, 1 \mathrm{H}), 7.20-7.37$ (m, $10 \mathrm{H}) .{ }^{13} \mathrm{C}-\mathrm{NMR}\left(\mathrm{CDCl}_{3}\right)$ 843.1, 51.1, 61.6, 117.5, 126.8, 127.0, 127.3, 128.1, 128.2, 128.3, 135.4, 140.6, 143.8; HRMS calcd for $\mathrm{C}_{17} \mathrm{H}_{19} \mathrm{~N} 237.1499$, Found 237.1483.

(2q)<smiles>C=CCC(NCCC)c1ccccc1</smiles>

J. Wang, Y. Zhang, W. Bao, Synth.Commun. 1996, 26, 2473-2478.

colorless wax; IR (neat) $3325 \mathrm{~cm}^{-1}$; ${ }^{1} \mathrm{H}-\mathrm{NMR}\left(\mathrm{CDCl}_{3}\right) \delta 0.85$ (t, $\left.J=7.32 \mathrm{~Hz}, 3 \mathrm{H}\right), 1.36-1.55$ (m, 3H), 2.31-2.46 (m, 4H), $3.64(\mathrm{t}, J=6.83 \mathrm{~Hz}, 1 \mathrm{H}), 5.01-5.14(\mathrm{~m}, 2 \mathrm{H}), 5.62-5.82(\mathrm{~m}, 1 \mathrm{H}), 7.16-7.40(\mathrm{~m}, 5 \mathrm{H})$. ${ }^{13} \mathrm{C}-\mathrm{NMR}\left(\mathrm{CDCl}_{3}\right) \delta 11.8,23.3,43.1,49.6,62.6,117.2,126.8,127.1,128.2,135.5,144.2$; HRMS calcd for $\mathrm{C}_{13} \mathrm{H}_{19} \mathrm{~N}$ 189.1517: Found $190.1594\left(\mathrm{Cl},\left(\mathrm{M}^{+}\right)+1\right)$; Anal calcd for $\mathrm{C}_{13} \mathrm{H}_{19} \mathrm{~N}$ : C, 82.48, H, 10.12, N, 7.20. Found C, 82.20, H, 10.00, N, 7.39.

(2r)<smiles>C=CCC(NC(C)c1ccccc1)c1ccccc1</smiles>

G. Alvaroe, C. Boga, D. Savoia, A. Umani-Ronchi, J. Chem. Soc. Perkin Trans.1. 1996, 875.

IR (neat) $3325 \mathrm{~cm}^{-1}$; ${ }^{1} \mathrm{H}-\mathrm{NMR}\left(\mathrm{CDCl}_{3}\right)$ (syn) $\delta 1.32(\mathrm{~d}, J=6.35 \mathrm{~Hz}, 1 \mathrm{H}), 1.65$ (brs, $\left.1 \mathrm{H}\right), 2.26-2.54(2$ H), 3.65-6.81 (m, 2H), 4.94-5.10 (m, 2H), 5.52-5.76 (m, 1H), 7.10-7.36 (m, 10H). (anti) $\delta 1.26(\mathrm{~d}, J=$ $6.83 \mathrm{~Hz}, 1 \mathrm{H}), 1.65$ (brs, 1H), 2.26-2.54 (2 H), 3.37 (t, J=3.07 Hz, 1H), 3.48 (q, J=6.60 Hz, 1H), 4.94$5.10(\mathrm{~m}, 2 \mathrm{H}), 5.52-5.76(\mathrm{~m}, 1 \mathrm{H}), 7.10-7.36(\mathrm{~m}, 10 \mathrm{H}) .{ }^{13} \mathrm{C}-\mathrm{NMR}\left(\mathrm{CDCl}_{3}\right)($ syn) $\delta$ 22.5, 42.1, 54.5, 59.6, 117.1, 126.5-128.3(6C), 135.3, 143.8, 146.0. (anti) 24.9, 43.3, 54.8, 58.97, 117.3, 126.5-128.3(6C), 135.6, 144.1, 145.6.; HRMS calcd for $\mathrm{C}_{18} \mathrm{H}_{21} \mathrm{~N}$ 251.1674: Found 252.1739(Cl,( $\left.\left.\mathrm{M}^{+}\right)+1\right)$. 
(2s)<smiles>C=CCC(NCCC)C1CCCCC1</smiles>

bp $50^{\circ} \mathrm{C} / 1 \mathrm{mmHg}$; IR (neat) $3325 \mathrm{~cm}^{-1} ;{ }^{1} \mathrm{H}-\mathrm{NMR}\left(\mathrm{CDCl}_{3}\right) \delta 0.90$ (t, $\left.J=7.08 \mathrm{~Hz}, 3 \mathrm{H}\right), 0.84-1.84$ (m, $13 \mathrm{H}), 1.98-2.13(\mathrm{~m}, 1 \mathrm{H}), 2.46-2.58(\mathrm{~m}, 2 \mathrm{H}), 2.52(\mathrm{t}, J=7.08 \mathrm{~Hz}, 2 \mathrm{H}), 5.01-5.13(\mathrm{~m}, 2 \mathrm{H}), 5.69-5.88(\mathrm{~m}$, 1H). ${ }^{13} \mathrm{C}-\mathrm{NMR}\left(\mathrm{CDCl}_{3}\right) \delta 11.9,23.6,26.8(3 \mathrm{H}), 28.9,29.5,35.7,40.9,50.1,62.1,116.5,137.0$; HRMS calcd for $\mathrm{C}_{13} \mathrm{H}_{25} \mathrm{~N}$ 195.1987: Found 196.2071(CI, $\left.\left(\mathrm{M}^{+}\right)+1\right)$.

(2t)

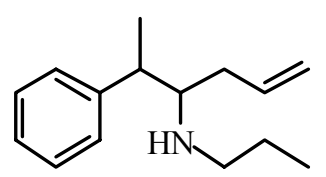

colorless wax; IR(neat) $3333 \mathrm{~cm}^{-1} ;{ }^{1} \mathrm{H}-\mathrm{NMR}\left(\mathrm{CDCl}_{3}\right.$ ) (major isomer) $\delta 0.85$ (t, $\left.J=7.32 \mathrm{~Hz}, 3 \mathrm{H}\right), 0.98$ (brs, $1 \mathrm{H}), 1.30(\mathrm{~d}, J=6.84 \mathrm{~Hz}, 3 \mathrm{H}), 1.21-1.52(\mathrm{~m}, 2 \mathrm{H}), 1.85-2.00(\mathrm{~m}, 1 \mathrm{H}), 2.05-2.20(\mathrm{~m}, 1 \mathrm{H}), 2.40-2.60$ (m, 2H), 2.60-2.75 (m, 1H), 2.80-2.90 (m, 1H), 5.01-5.14 (m, 2H), 5.62-5.82 (m, 1H), 7.16-7.40 (m, 5H). ${ }^{13} \mathrm{C}-\mathrm{NMR}\left(\mathrm{CDCl}_{3}\right) \delta 11.8,17.2,23.5,36.3,42.8,49.6,62.8,116.7,126.0,127.8,128.1,136.1,145.3$; HRMS calcd for $\mathrm{C}_{15} \mathrm{H}_{23} \mathrm{~N}$ 217.1830: Found d217.1826; Anal calcd for $\mathrm{C}_{15} \mathrm{H}_{23} \mathrm{~N}$ : C, 82.48, H, 10.12, N, 7.20. Found C, 82.20, H, 10.00, N, 7.39.

(2u)

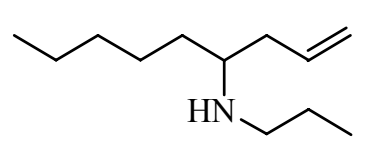

bp $80^{\circ} \mathrm{C} / 0.3 \mathrm{mmHg}$; IR(neat) $2931 \mathrm{~cm}^{-1}$; ${ }^{1} \mathrm{H}-\mathrm{NMR}\left(\mathrm{CDCl}_{3}\right) \delta 0.86-0.93(\mathrm{~m}, 6 \mathrm{H}), 1.29-1.54(\mathrm{~m}, 11 \mathrm{H})$, 2.05-2.28 (m, 2H), 2.48-2.57 (m, 3H), 5.04-5.10 (m, 2H), 5.70-5.86 (m, 1H); ${ }^{13} \mathrm{C}-\mathrm{NMR}\left(\mathrm{CDCl}_{3}\right)$ 812.4, 14.6, 23.1, 23.9, 26.0, 32.6, 34.5, 39.0, 49.6, 57.4, 117.4, 136.4; HRMS calcd for $\mathrm{C}_{12} \mathrm{H}_{25} \mathrm{~N}$ 183.1987: Found. $184.2075\left(\mathrm{CI},\left(\mathrm{M}^{+}\right)+1\right)$. 
3. ${ }^{13} \mathrm{C}$ NMR Chart
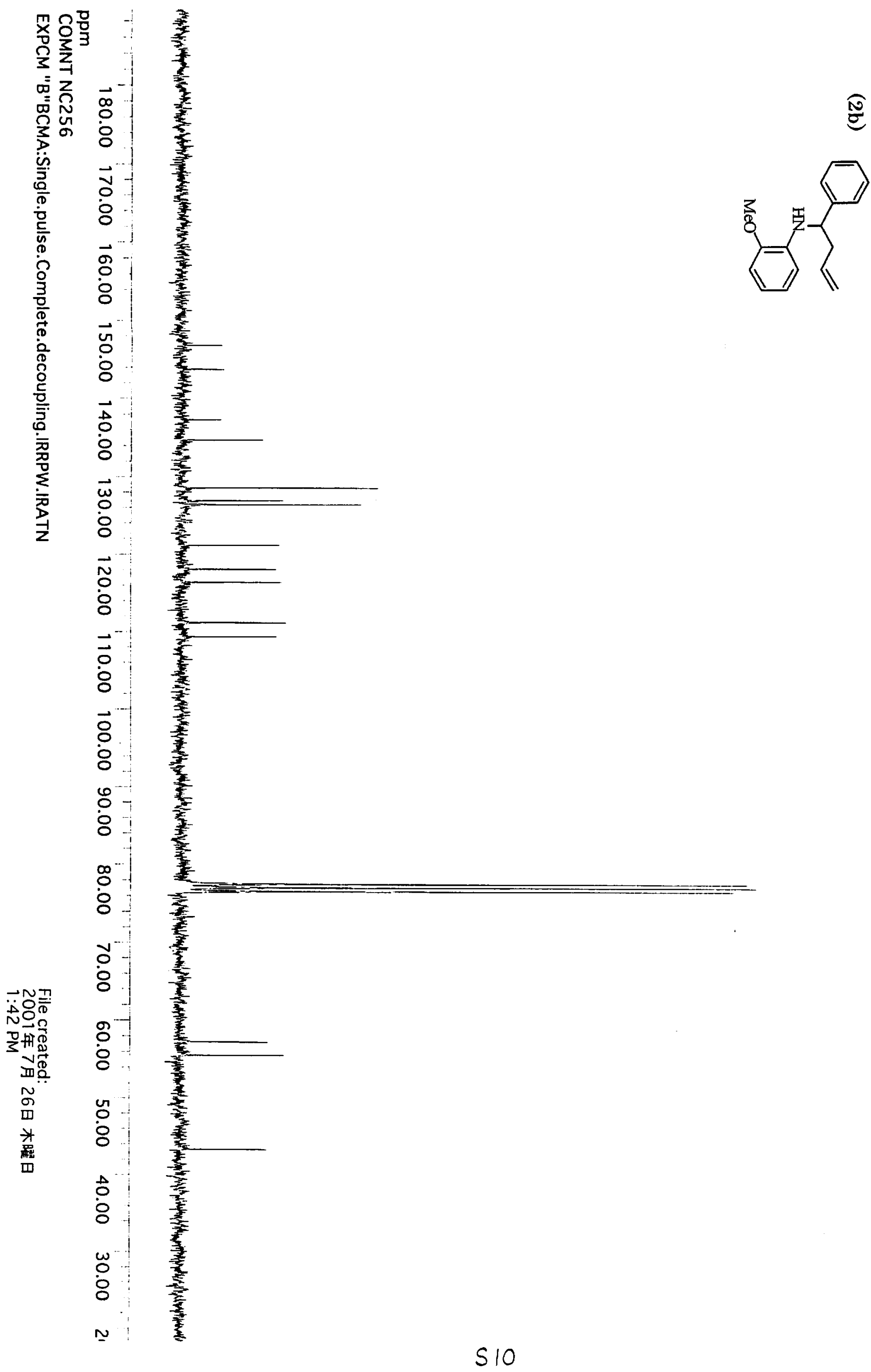


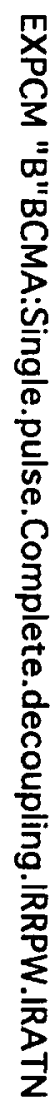

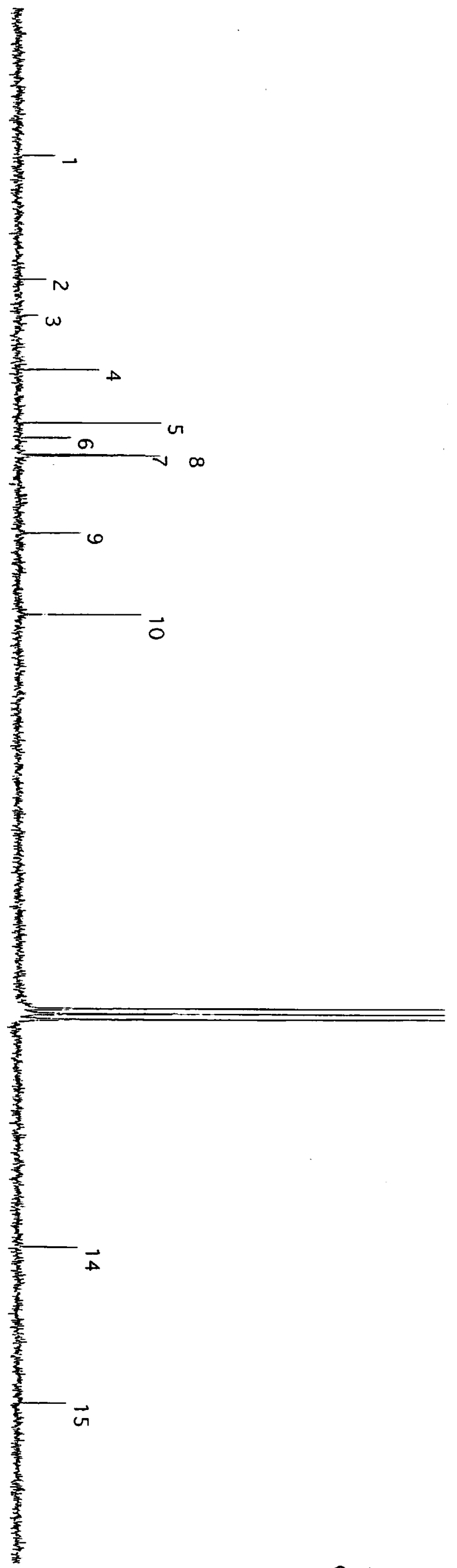

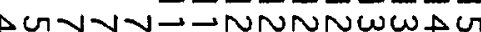

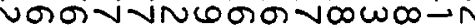

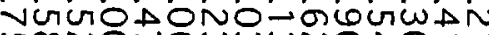

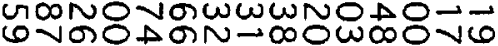

NWGiven V000000000600

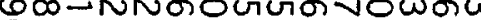

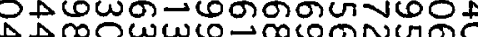

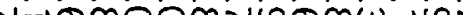
uncviooogaw vow

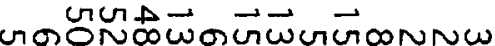
NWG

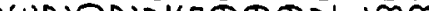

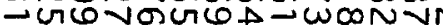
Vova - V VONGOD

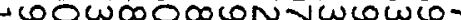
inivinirg

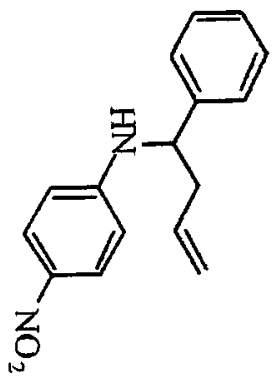




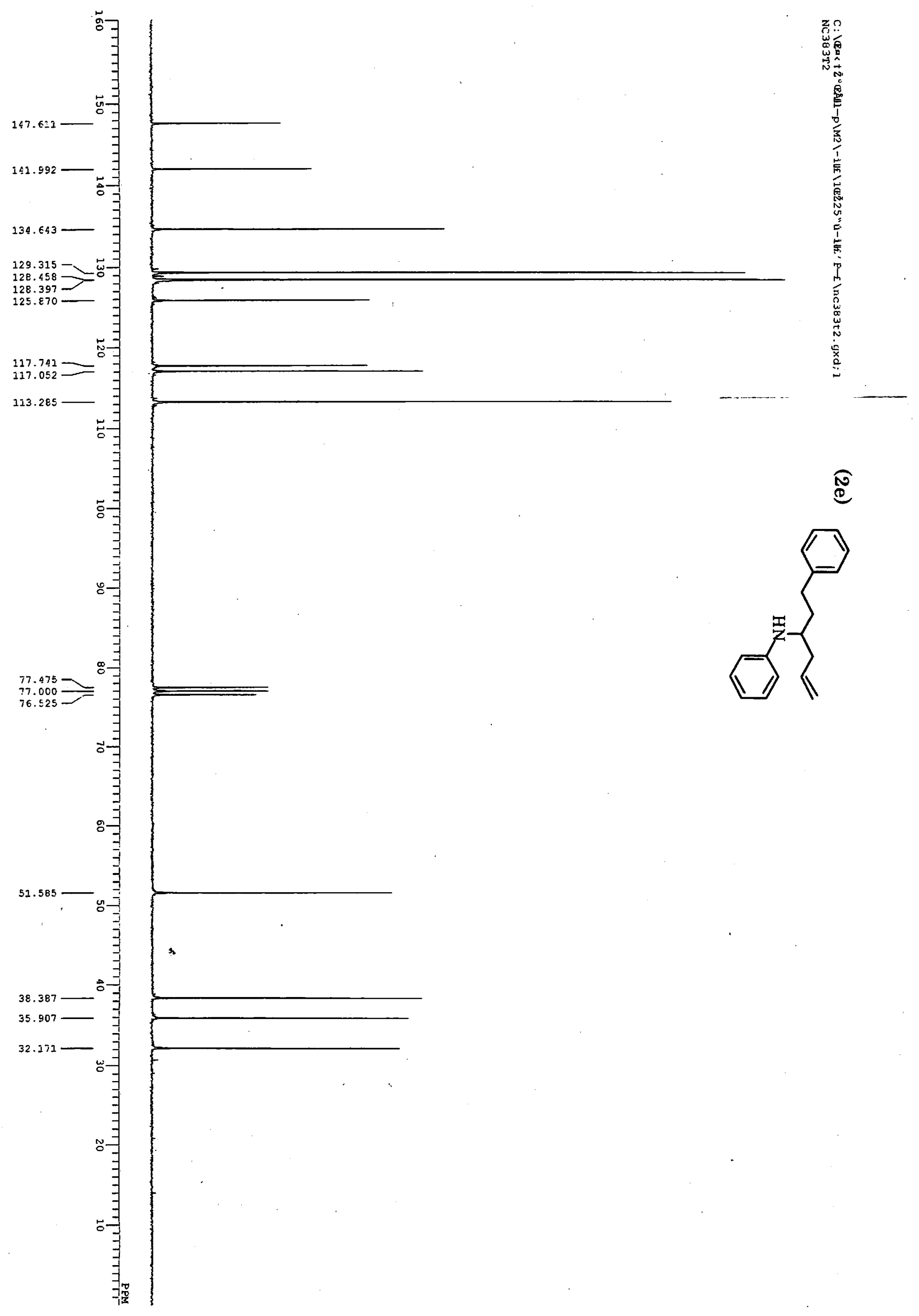




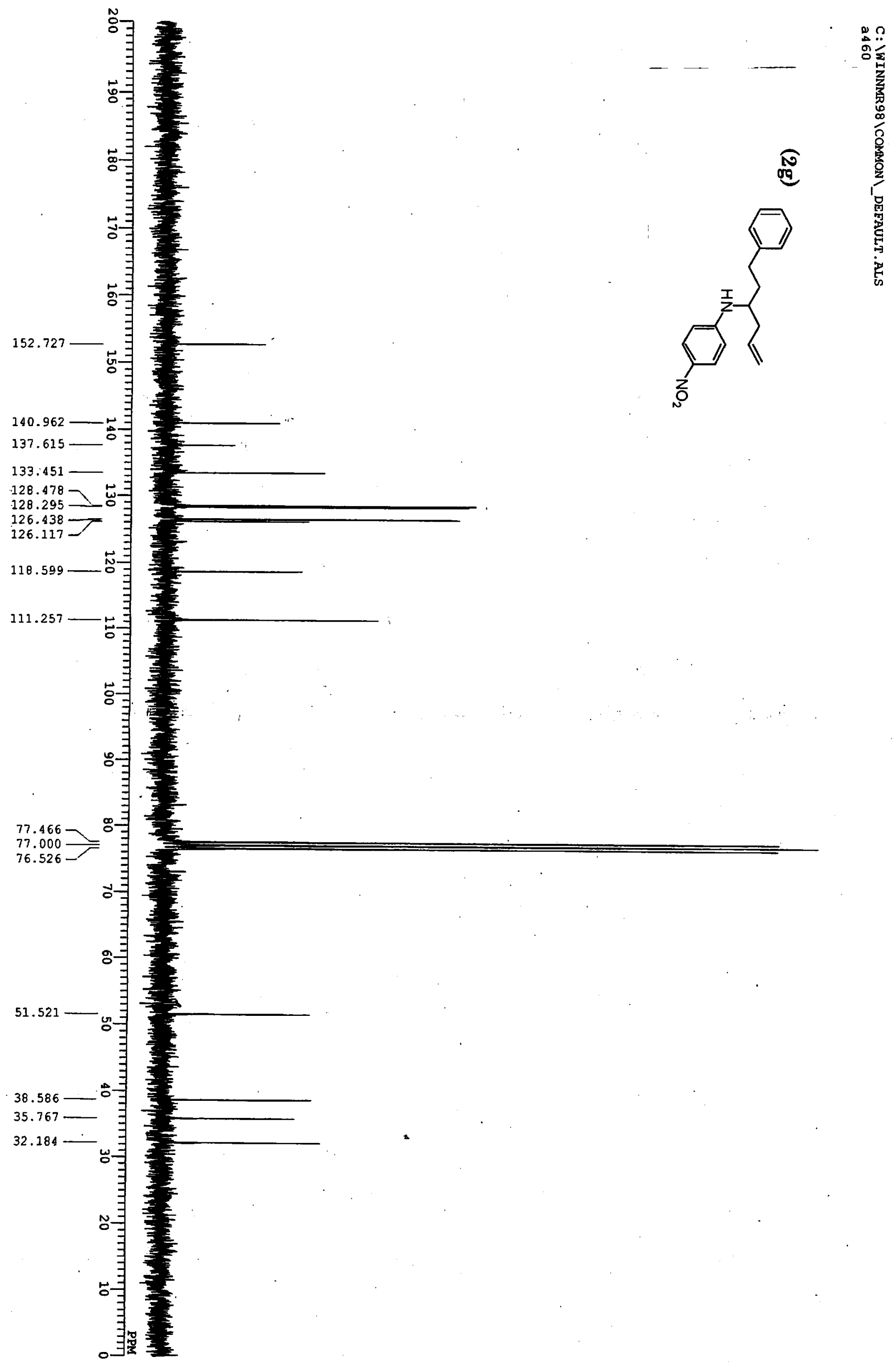




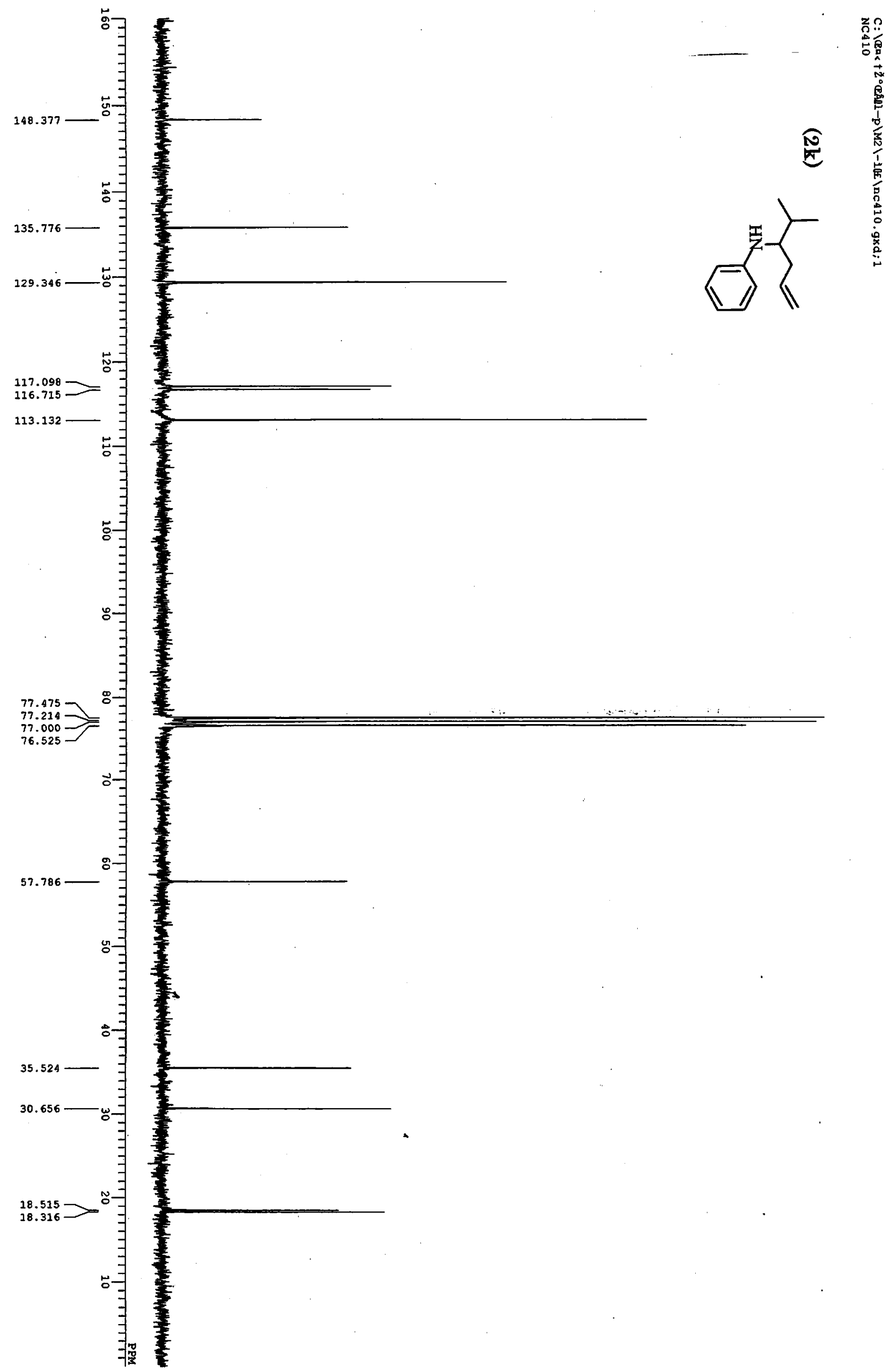




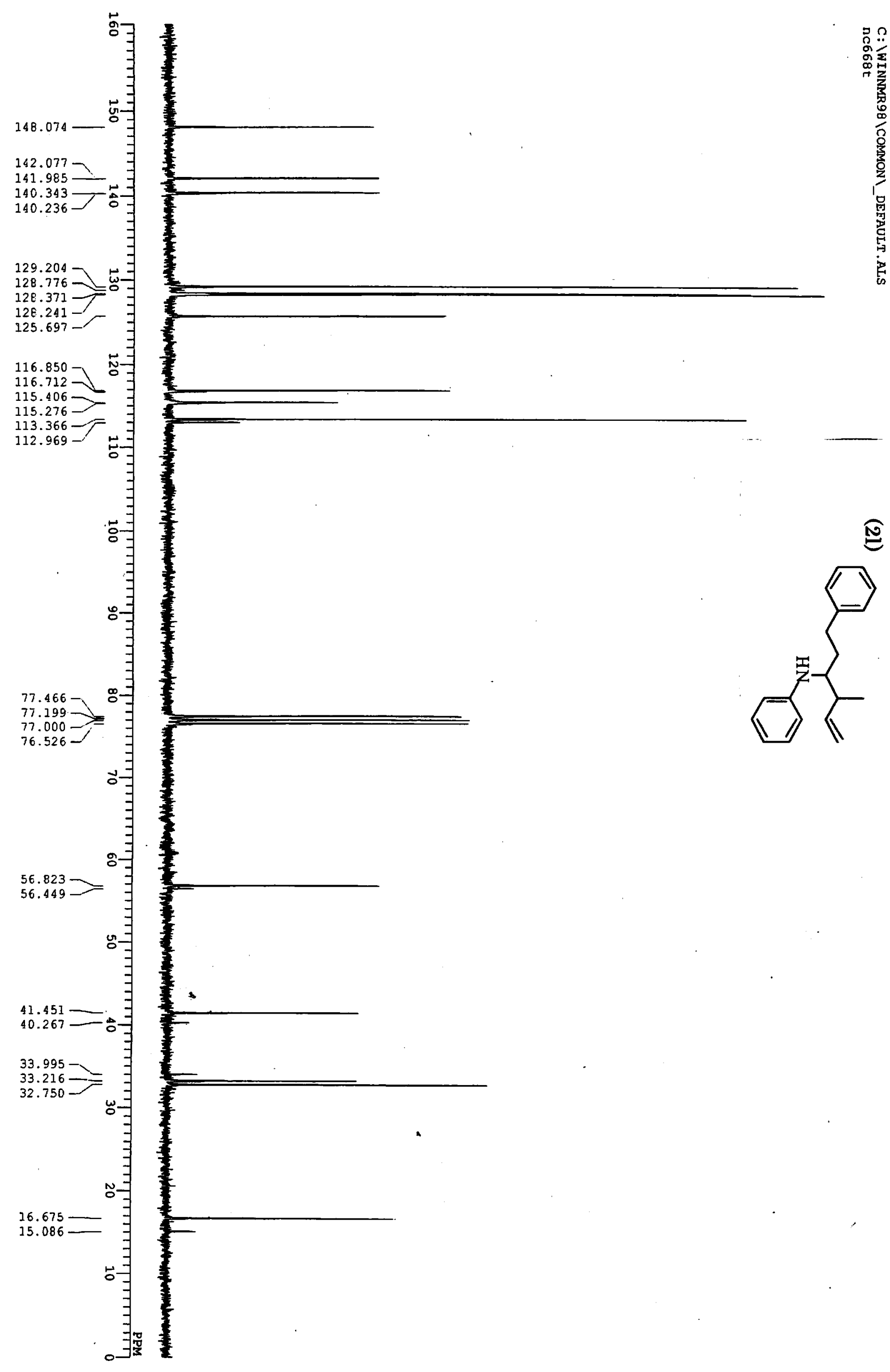




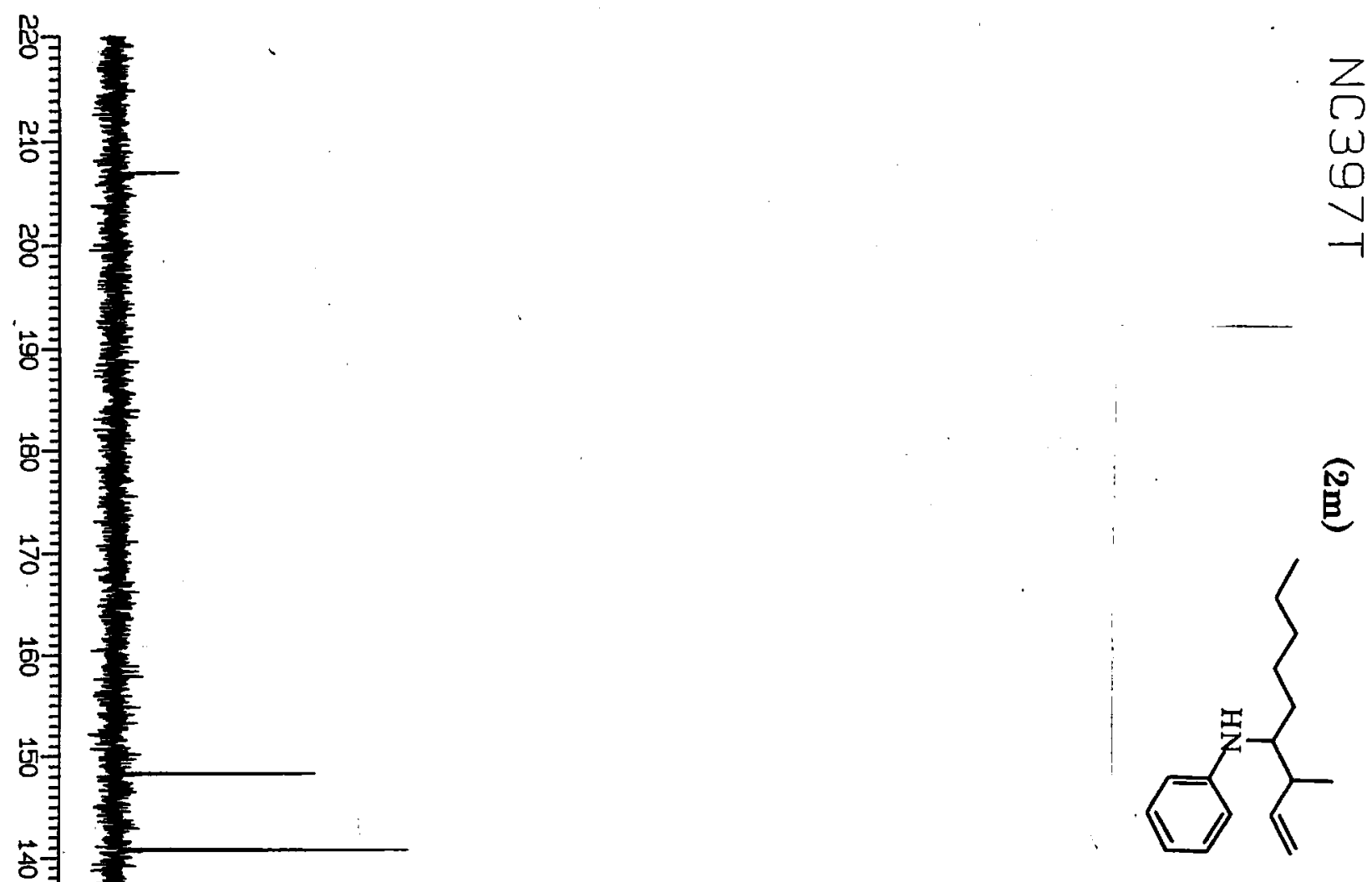

N

客

安筆

果

品丰

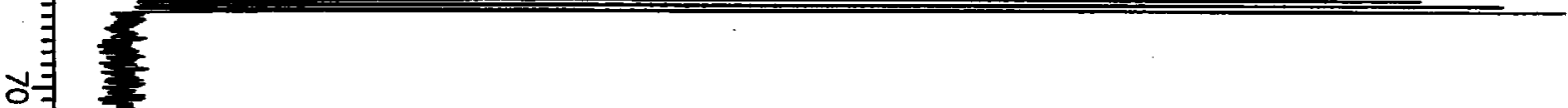

它录

目

宫

8

봉킄

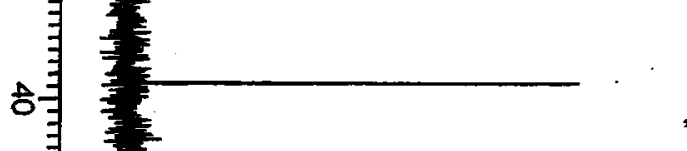

幽

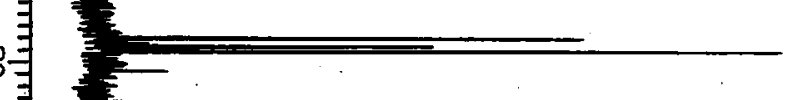

ㅋ.

역

台手

埾 


$$
E
$$




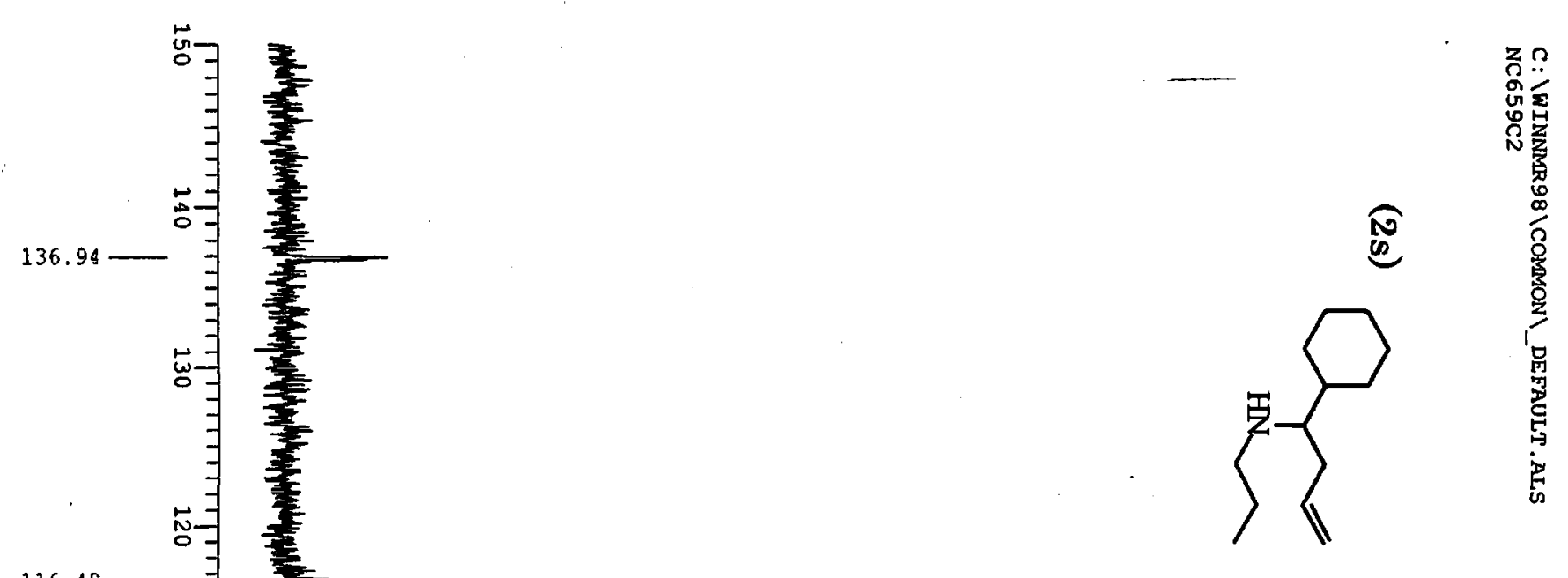

136.46
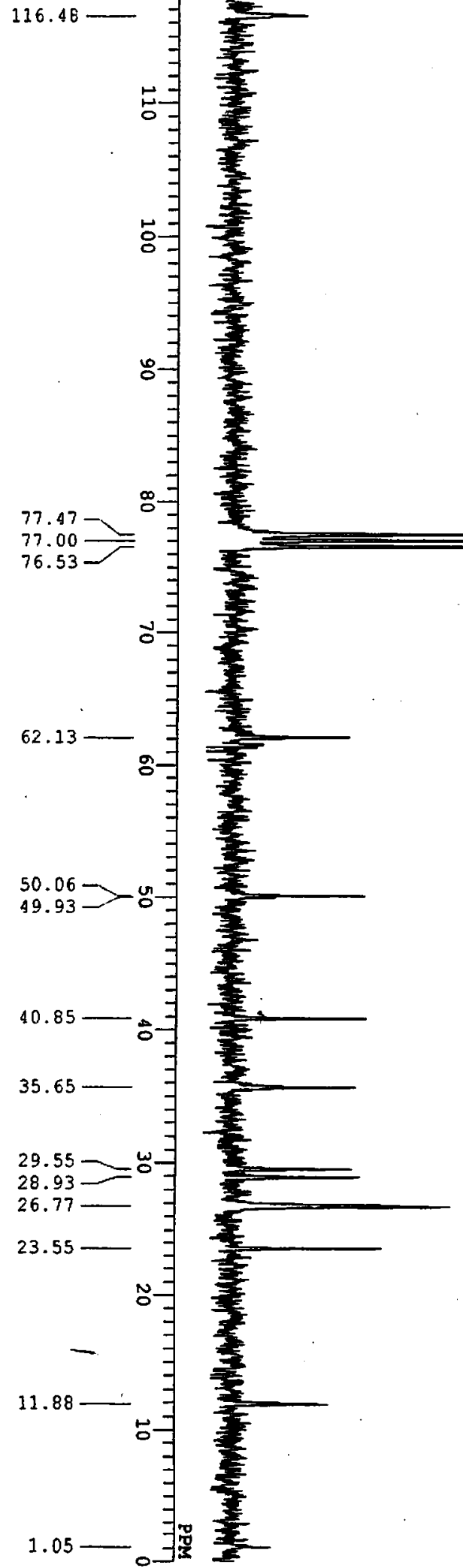


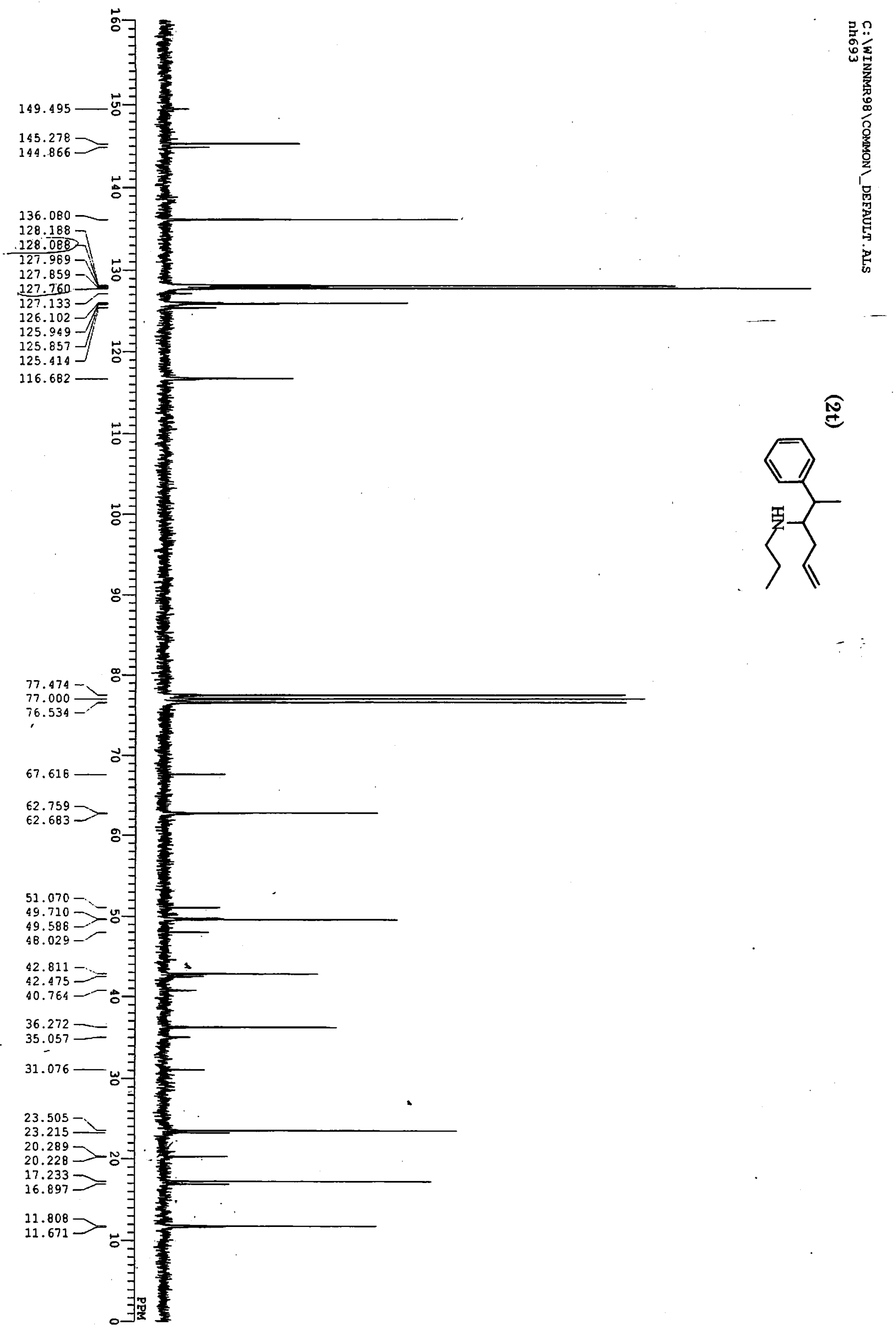




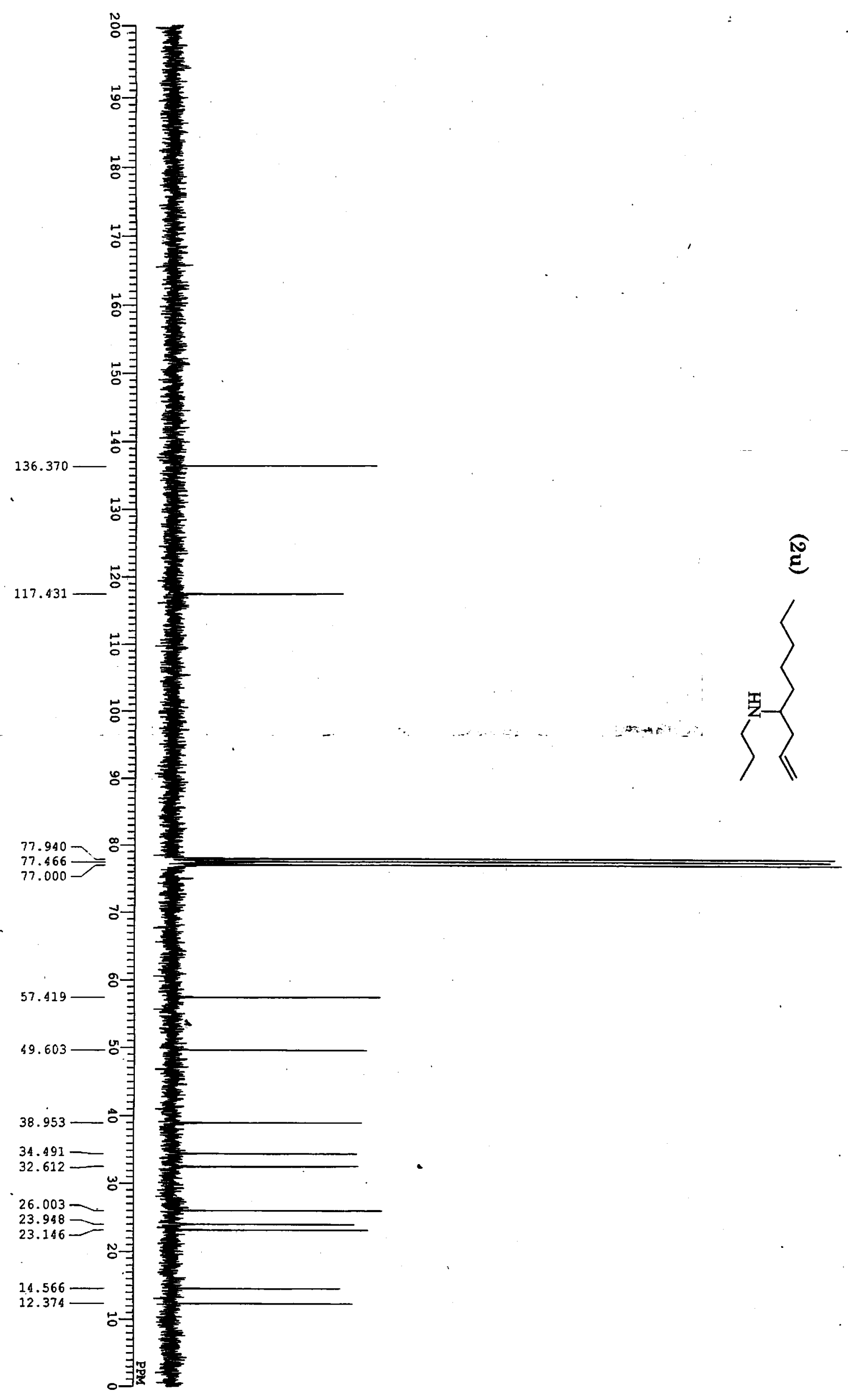

